\title{
Erratum: Hacking the Quantum Key Distribution System by Exploiting the Avalanche-Transition Region of Single-Photon Detectors [Phys. Rev. Applied 10, 064062 (2018)]
}

\author{
Yong-Jun Qian $\odot$, De-Yong He, Shuang Wang, ${ }^{*}$ Wei Chen, Zhen-Qiang Yin, Guang-Can Guo, and \\ Zheng-Fu Han
}

(Q) (Received 27 February 2020; accepted 9 March 2020; published 20 March 2020)

DOI: 10.1103/PhysRevApplied.13.039901

Since we found that the decoy-state method had been proposed by several research groups, the following reference [Phys. Rev. Lett. 94, 230503 (2005)] should be added at the end of the paragraph 2 in our paper: "The existence of multiphoton states gave Eve a chink to implement a photon- number-splitting attack until the decoy-state method was proposed $[8,9,29,30,46] . "$

[46] X. B. Wang, Beating the Photon-Number-Splitting Attack in Practical Quantum Cryptography, Phys. Rev. Lett. 94, 230503 (2005).

\footnotetext{
*wshuang@ustc.edu.cn
} 